\title{
An Atom Laser over Optical Lasers
}

\author{
Arbind Kumar Sah \\ Department of Science and Humanities, Pashimanchal Campus, Lamachaur, Pokhara \\ Email: aksphy@yahoo.com
}

\begin{abstract}
An atom laser is a coherent state of propagating atoms. They are created out of a Bose Einstein Condensation (BEC) of atoms which are output coupled using various techniques. An optical laser or conventional laser generates a coherent beam of light waves where as an atom laser produces a coherent beam of matter waves. An atom laser will have a major impact on the fields of atom optics, atom lithography and precision measurements.
\end{abstract}

Keywords: BEC, atomic optics, atom lithography and atom interferometry.

\section{INTRODUCTION}

The Technology which we use nowadays are all electronic which contain electronic components like resistors, capacitors, inductors, logic gates, etc. and latter development integrated circuits called IC. The development of IC chips reduces the sizes of electronic devices in the smaller forms such like laptops, faster computers; mobiles, etc. while they were in large size previous as electronic components are used individually. Laser is one of the photonic device where photons play the role same as that played by electrons in electronics. Optical signals have larger bandwidth than electrical signals and therefore, can accommodate a large number of channels per a given volume. The optical laser and an atom laser both are photonic technology but there are differences in their uses. In holography, optical laser uses the diffraction of a photon beam whereas an atom laser uses the diffraction of atoms. ${ }^{5,6}$

\section{MATERIAL AND METHODS}

Indeed, "laser' stands for "Light Amplification by Stimulated Emission of Relation" which is not particularly related to the particular objects called an atom laser. An atom lager is a device in which atom plays the same role as the photon does in the case of an optical lager. Such laser produces a coherent beam of matter waves and each atom is associated with a de-Broglie wave. When a dilute gas is cooled to temperature near to zero, the de-Broglie waves are merged together to form a coherent matter waves. Therefore,an atom laser requires thermal cloud of ultra-cold atoms as an active medium. Atoms are to be cooled to nano-kelvin temperatures, when they form BEC \& create the state of coherent matter waves. The discovery of BEC has heralded the invention of atom laser on the fields of atomic optics, atom holography \& precision measurement are discussed here. ${ }^{1,2}$

The first atom laser was demonstrated at Massachusetts Institue of Technology (MIT) by professor Wolfyong Kotterle et. al. in November 1996. from the creation of the atom laser, there has been surge in the recreation of atom lasers there has been surge in the recreation of atom laser along with different techniques for output coupling and in general research. The current development stage of the atom laser is analogous to that of the optical laser during its discovery in the 1960 . To that effect, the equipment and techniques are in their earliest developmental phases \& still strictly in the domain of research laboratories.

\section{DISCUSSIONS:}

\subsection{Physics:}

In 1924, the Indian physicist SatyendraNath Bose derived the Planck's law for back-body radiation by treating the photons as a gas of identical particles. Einstein generalized Bose's theory to other particles. The theory is now known as Bose-Einstein Statics \& particles are called bosons. Einstein predicted that at sufficiently low temperatures, all the atoms in a ideal gas of identical atoms might be locked together in a 
single lowest quantum state of the system, which is called BEC. The BEC of gas involves two steps:

\subsubsection{Laser Doppler Cooling}

In this step the atoms are cooled at low density to milli-kelvin temps by the action of laser beam of approximate frequency. Actually, Doppler cooling is a mechanism that can be used to trap and cool the atoms.
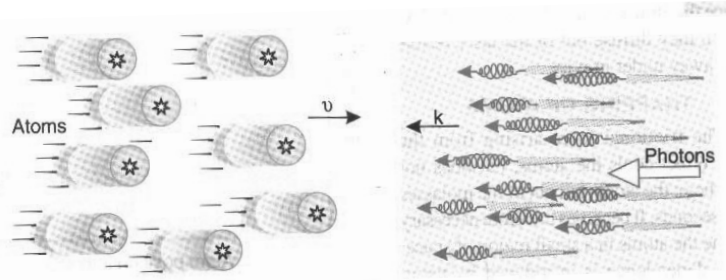

Fig. 1 A stream of atoms colliding with a laserbeam in the process of laser cooling

In practice six laser beams(two counter propagating beams each, one along the positive direction \& the other along the negative direction) \& a pair of magnetic field coils forming a magneto-optical trap(MOT) can cool more than a billion atoms to micro-kelvin- tempratures.

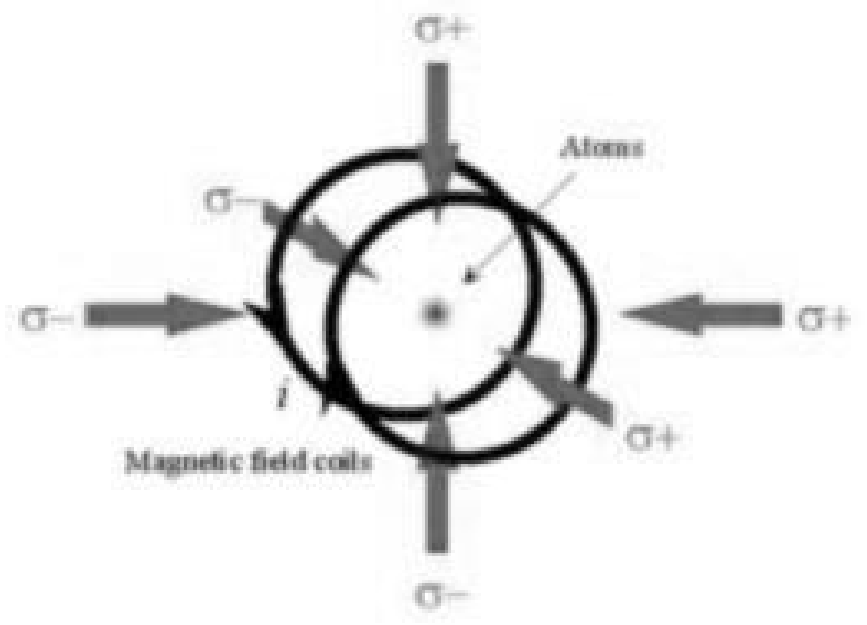

Fig. 2 Magneto-Optical Trap

\subsubsection{Evaporative Cooling:}

The atoms are further cooled to ultra-cold temps using the technique of evaporative cooling. The atoms are cooled first in the presence of the magnetooptic trap. Then, the laser beams are turned off and a magnetic field of the same type but much stronger than the used in MOT is applied to perform the role of a bowl and confine the cold atoms. Continuing to cool involves continuously lowering the sides of the magnetic trap. The process is halted when the temp goes down to nearly $100 \mathrm{nk}$ and only a few atoms are left to form a condensate.

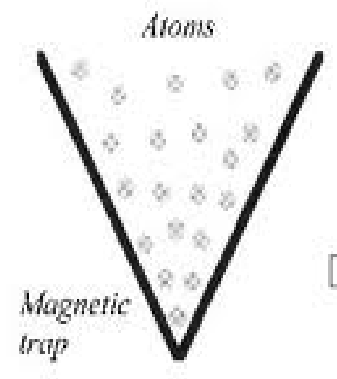

(a)

Atoms held in a magnetic trap

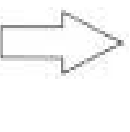

Cooling works by slowly lowering the sides to release the hot atoms at top

Fig. 3

\subsection{Applications:}

Atom lasers may have other measurement of fundamental standards together with three majors, which are discussed here:

\subsubsection{Holography:}

An atom laser is critical for atom holography. Similar to conventional holography uses the diffraction of a photon beam, atom holography uses the diffraction of atoms. As the De-Broglie wavelength of atoms is much samaller than the wavelength of light, so atom laser can create much higher resolution holography 3-D image. Atom holography might be used to project complex integrated circuit patterns, just a few nanometres in scale, onto semi-conductors.

\subsubsection{Atom Interferrometry:}

Another application of an atom laser is in the atom interferrometry. In an atom interferrometer, an atomic wave packet is coherently split into two packets that follow different paths before recombining. Atom, interferrometers, which can be more sensitive than optical interferrometers, could be used to test quantum theory, and have such high precision that they may even be able to detect changes in space- 
time. This is because the De-Brogile wavelength of the atoms is much smaller than the wavelength of light, and the atoms have mass. Atom laser would allow the use of devices like Michelsion interferrometres, precision atomic clocks, etc.

\subsubsection{Atom Optics:}

The third specific application of the atom laser goes towards the non-linear optics Until recently, most atom optics experiments could be thought of as single particle phenomena supposing negligence of interactions between particles. In conventional non-linear optics, photons interact with each other through some mediating materials such as a transparent crystal. A common non-linear optical phenomenon is "four-wave mixing". The exchange of energy and momentum between the waves, mediated by non-linear-crystal, results in the production of fourth wave frequency as fig. (IV $)^{4}$ a

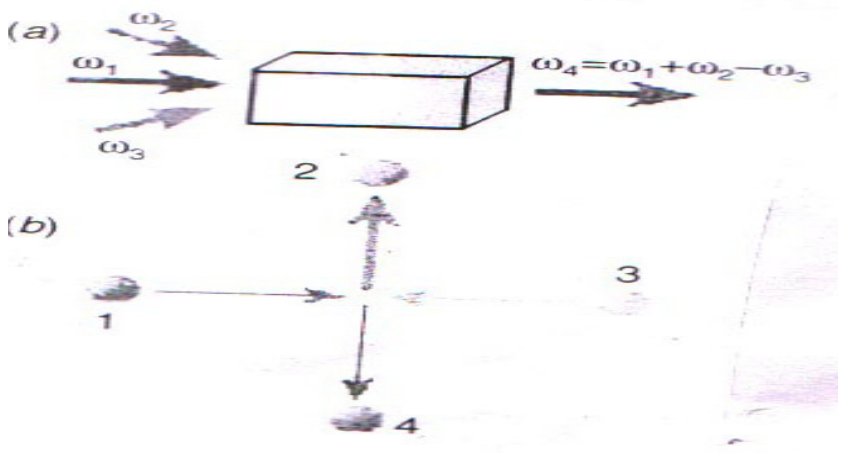

Fig. 4 Four- wave mixing

In 1998, an analogous process with matter waves was predicted. The four wave mixing process arises from collision between pairs of atoms ( $1 \& 2$ in fig. IVb). One pair of atoms scatters in the direction of third, incident matter wave beam and amplifies it (3).By the conservation of energy \& momentum, the other pair of atoms produces a fourth separate beam (4).

\section{CHARACTERISTICS:}

An atom laser consists of similar principal parts of analogous to conventional lasers but in different ways. Active medium is a thermal ultra-cold atom. Evaporative cooling plays the role of pumping.
Analogous to conventional laser as the optical cavity formed by the mirrors, the cavity resonator is magnetic trap here. The process of condensation of the atoms into the ground state of a magnetic trap is the stimulated emission into a single mode. A beam of atoms (i.e. matter waves) is the Output of an atom laser. Optical laser radiate several modes while an atom laser operates only single mode. Population inversion is essential in the conventional laser whereas that doesn't occur in this case.

\section{CONCLUSION:}

Important features of a laser beam are monochromaticity, coherence, intensity and directionality of the beam of light. If these properties can be maintained when atoms are released from the condensation, theywill be ofhigherdegreeandquality.

Therefore, the conventional laser is aimed to next generation whereas an atom laser containing matter waves instead of electromagnetic waves for further next generation.

\section{REFERENCES:}

[1] Abdeldayen, H. Frazier, DO Palley; with erow, W.K, 2009, Recent Advances in Photonics Devices for Optical Computing, NASA space science Laboratory, USA.

[2] Keiser, Gerd, Optical Fibre Communication, Boston University, National Taiwan University of Science \& Technology.

[3] Lamichhane, Tika Ram, \& Bhat, Mahesh Datt, 2006 B.S., A text Book of engineering Physics, Sunlight Publication, Kathmandu, Nepal.

[4] Sapkota, Bal Krishan, Pokhrel, Bhadra \& Bhatttarai, Binod Kumar, 2067 Fundamental of Eng. Phys/ first edition, phys instruction Committee, Pulchowk, Nepal

[5] Subramanian, N \& Lal, Brij, 2006, A text book of Optics, Multi-color edition, S.Chand Publication. 